\title{
Interactive comment on "Physical constraints for respiration in microbial hotspots in soil and their importance for denitrification" by Steffen Schlüter et al.
}

\section{Steffen Schlüter et al.}

steffen.schlueter@ufz.de

Received and published: 20 June 2019

We are grateful to Ali Ebrahimi for his constructive suggestions to improve the quality of the paper. In the following we reply as comprehensively as possible to each of the issues raised:

l) Some of experimental decisions require better justifications in the context of denitrification process in natural soil systems. For instance, why two isolates with full or partial denitrification steps are chosen? I could think of this choice as an interesting option when studying the residence times of $\mathrm{N2O}$ in soil aggregates but I was confused with lack of justifications for these choices. A similar problem could be stated to the

Printer-friendly version

Discussion paper 
design of layered vs. random microbial hotspots "aggregates". It was nearly impossible to conceptualize how these types of distributions might actually have any relevance for soil systems or representing any specific scenarios with generalizable outcome. In the present form it is quite confusing and hard to understand what is the relevant, unknown question that the study aims to address and how the experimental design helps to navigate in that direction. The conclusions are mostly based on describing the observations and lacks discussion and interpretations to link these specific results to advance general knowledge on soil denitrification /respiration. My understanding is that a simple mathematical model that would explain the basic mechanisms underlying the observations could go a long way to translate the results into general conclusions.

The rationale for using two functionally different denitrifier pure cultures is threefold: 1. The regulatory phenotypes of P. denitrificans and A. tumefaciens are well known and result in characteristic and reproducible gaseous product stoichiometries as described in section 3.1 (Lines- 200-217). Knowing growth and product kinetics in unconstrained pellets beforehand (section 3.1 - experiment without sand matrix), our aim was to deduce the activity and interaction of functionally distinct hotspots in the sand experiments from overall gas kinetics, and collate them with the experimental conditions (hotspot architecture and saturation); 2. Unlike for natural denitrifying consortia, the growth parameters of the two strains are known for a range of conditions (temperature, $\mathrm{pH}$, oxidant to reductant ratios, etc) and the outcome of our experiment thus lends itself to 3D transport-reaction modelling explicit for microbial hotspots in a follow up study. 3 . A reductionist approach is helpful to advance process understanding before moving to complex dynamics in real soil systems (Rillig and Antonovics, 2019). We have rewritten parts of the introduction, and hope to have clarified the rational of our experimental setup.

Printer-friendly version

Discussion paper

A lack of justification for the two different hotspot architectures was also mentioned by reviewer 1 . Having the inoculated glass pellets as experimental unit, it was our intention to create contrasting hotspot architectures with respect to local $\mathrm{O} 2$ consumption 
and gaseous transport of $\mathrm{NO}$ and $\mathrm{N} 2 \mathrm{O}$ between the functionally distinct hotspots. For this we chose a setup with minimal distance between hotspots (layered) and one with maximum distance (random) between the hotspots. In addition, the layered design gave us the option to place the functionally different strains into individual layers, with the complete denitrifier P. denitrificans either on top or in the bottom of the jar. This indeed enabled us to study the effect of functionally and spatially distinct hotspots on the residence time and fate of $\mathrm{N} 2 \mathrm{O}$ (L. 390-395). We are aware that such a separation of different strains can hardly be found in natural soil. However, only such an artificial but well-defined spatial arrangement allows us to separate the effects of local gas consumption/production from those of diffusion. In this way it is possible to connect the macroscopic concept of hotspots which is around since quite a while with microscopic understanding of denitrification processes the pore scale. Our experimental design is motivated to allow for this connection following a reductionist approach. This is now explained in more detail in the Introduction section.

We agree that the overall research question the study aims to address has not been properly carved out in the original version of the paper, but will be clarified in the new version of the manuscript. The "hotspot" concept has been around for decades (since (Parkin, 1987)), and we urgently need experimental model systems of hotspots to validate pore-scale models of denitrification, which, in turn, are needed to develop NO and $\mathrm{N} 2 \mathrm{O}$ mitigation strategies. That this is - necessarily - reductionist, we have mentioned above.

II) While authors attempted to justify the choice of closed system for their experiments, but there are fundamental issues to measure gas fluxes in the closed system. One of the main issues is that the accumulation of gases in the headspace for a long time will result into altering dissolved gas concentration profile within the soil aggregates and the soil profile itself that will ultimately affect the gas fluxes from the soil surface. From the theoretical calculations (unpublished, but shareable upon request), having even quite large headspace (1litter) would affect gas concentration profile and gas storage

Printer-friendly version

Discussion paper

. 
in soil within less than 10 hours. In other studies to resolve this issue, partial closing of the headspace is suggested (A. Ebrahimi and Or 2018). This is especially important to make sure that the oxygen concentration at the headspace remains unchanged, similar to natural soil profile throughout the experiments. In the current study, it is hard to disentangle the main mechanisms of forming anoxic microsites in the soil profile, if the main reason is because of the physical restrictions and presence of soil aggregates or just simply because of oxygen consumption and lack of oxygen in the headspace.

It was never our intention to study respiration/denitrification kinetics at unchanged "steady-state" $\mathrm{O} 2$ conditions. Much to the contrary, we used batch incubation with a finite amount of $\mathrm{O} 2$ to gradually induce anoxia in the artificial hotspot system. All known heterotrophic denitrifiers are facultative aerobes, i.e. they thrive with $\mathrm{O} 2$ as electron acceptor. In the present experiment, we were particularly interested in the oxic-anoxic transition of denitrifiers, as it determines to which degree denitrification enzymes are induced. The extent to which and the sequence in which denitrification enzymes are induced in response to progressing anoxia determine denitrification enzyme kinetics and hence its product stoichiometry. This has its representation in the well-known fact that $\mathrm{N} 2 \mathrm{O}$ emissions are episodic, driven by rain falls and/or periods of high auto- or heterotrophic $\mathrm{O} 2$ consumptions as elaborated on in the discussion (L. 362-374). Our experiment conceptualizes this behavior by loading the hotspots with an easily degradable $\mathrm{C}$ medium together with growing denitrifiers and explicitly aims at studying the interplay between oxic and anoxic respiration and the effect of hotspot architecture and water saturation thereon. A welcome side effect of a static batch setup (as opposed to dynamic incubation at steady-state $\mathrm{O} 2$ levels) is that the former allows for sensitive detection of $\mathrm{N} 2$ at high temporal resolution, which appears justified given the fact that induction and functioning of $\mathrm{N} 2 \mathrm{O}$ reductase (here in $\mathrm{P}$. denitrificans) is instrumental for the understanding of $\mathrm{N} 2 \mathrm{O}$ emissions.

III) While I would like to be supportive of the idea of microbial hotspots, given the experimental design, it is hard to understand why these porous beads are hotspots

Printer-friendly version

Discussion paper
Interactive comment 
for the microbial activity, compared to the full sample (background sand). First, there is no explicit observations of microbial activity at the bead scale. Second, the choice of Sistrom's medium (rich, dissolved medium) insures that substrates are uniformly distributed all over the flask (sand+beads) and knowing the fact that bacterial cells could easy spread all over the flask in the given water contents ( $A$. N. Ebrahimi and Or 2014; Tecon and Or 2016), even if pre-inoculated only within the beads, I would then argue that the substrate and bacteria could actually be quite uniformly distributed in the flask and turns the whole system and not only beads into "microbial hotspots". This might still be fine, if the argument of "microbial hotspot" is not based on the substrate or bacteria distribution but rather physical regulations that beads impose on the oxygen gradients, leading to anoxic hotspots in the beads. However, this argument is also unlikely given the data presented in Figure 6 that shows similar air connectivity and tortuosity for the hotspots and the full system. Surprisingly, air distance seems to be higher in the full system (Figure 6C) that could mean some regions in the full system (likely the bottom of the flask) could be even more anoxic than the porous beads. I think this aspect of the research will require better explanations of the assumptions and the reasoning behind the experimental design.

We are fully aware that the dissolved carbon substrate and nitrate diffuses out of the glass beads into the sand and that at the lowest saturation (30\%WFPS) they have also been transported convectively by capillary forces. This was demonstrated with a separate brilliant blue experiment in the supporting information. The fact that oxygen consumption was slow at $30 \%$ WFPS is indirect evidence that the bacteria mainly remained in the hotspots. In other words, under well-aerated conditions only substrate limitation can explain a reduction in microbial growth as compared to the $60 \%$ WFPS and this can only occur if the substrates left the hotspots (highest loss of all saturations), but the cells didn't follow to the same degree but instead were nurtured more steadily via diffusion of substrates back into the hotspots. For the $60 \%$ and $90 \%$ WFPS case we can only speculate, as to how far bacterial dispersal is really relevant. A. tumefaciens is known to possess flagella (Merritt et al., 2007), but P. denitrificans is not

Printer-friendly version

Discussion paper
Interactive comment 
motile (personal communication with Linda Bergaust, NMBU, Norway). Experimental data on bacterial dispersal rates in soil is scarce. In a recent paper (Juyal et al., 2018), the first cells appeared in a distance of $15 \mathrm{~mm}$ after nine days (216h) for Bacillus subtilis and Pseudomonas fluorescens in repacked soils at similar bulk density $\left(1.5 \mathrm{~g} / \mathrm{cm}^{3}\right)$ and saturation (60\%). In our experiment, with the highest substrate concentration in the hotspots and most oxic-anoxic transitions already occurring after 3-6 days, the bottom of the jar might not have been colonized that quickly, but this cannot be backed by data. We will discuss the probability of bacterial spread at high saturations in the discussion now.

- In general, the Figures require more comprehensive captions. In the current form, it was painful to get full grasp of the figures without going back and forth into the text to learn the conditions that the experiments were performed.Minor concerns:

- In general, the Figures require more comprehensive captions. In the current form, it was painful to get full grasp of the figures without going back and forth into the text to learn the conditions that the experiments were performed.

A minimum amount of information on the incubation conditions is now added to the captions.

I think it would help a lot to use equal range for N2, NO and N2O or ploting the ratios of these gases to the total amount of available nitrogen. It was really hard to compare the rates of these gases to each other given the way the results were presented. Similar comment could be made for $\mathrm{O} 2$ and $\mathrm{CO} 2$.

We have now used the same range for $\mathrm{O} 2$ and $\mathrm{CO} 2$ and the same range for $\mathrm{NO}, \mathrm{N} 2 \mathrm{O}$ and N2 (on linear scale with logarithmic scale as an inset).

Throughout the manuscript, a few times the arguments were based on assuming that $P$. denitrificans is slow grower because it produces less CO2. However, this argu-

Printer-friendly version

Discussion paper (1) 
ment would only hold if both strains would have similar yield of converting substrate to biomass and CO2. Otherwise, one may argue $P$. dentrificans is more efficient on converting substrate to biomass and that is why produces less CO2.

Yes, this statement is shaky, when only based on CO2. However, it can be made when also taking the consumption of oxygen into account. This has been sated in lines 201-202.

- In Figure 4, scenario with 30 percent WFPS starts with about 200umol more 02 compared to 90 percent WFPS, however at the end of the experiment both scenario produces approximately 450umol CO2 with no O2 left in the flask. I was wondering where does the extra $\mathrm{O} 2$ is gone in 30 percent WFPS scenario? It might help to check again the mass conservations for different elements.

The values represent the amount of gaseous oxygen in the flask. In the 90\% WFPS there is less gaseous oxygen but more dissolved oxygen to start with, as more of the pore space is occupied by water. More specifically, the internal flask volume amounts to $238.3 \mathrm{ml}$ of which $118.3 \mathrm{ml}$ is occupied by the total pore volume including the headspace and the porosity of the sand. The liquid volume (including the liquid stored in glass beads) in the 90\% WFPS and 30\% WFPS treatments amounts to $49.6 \mathrm{ml}$ and $20.8 \mathrm{ml}$, respectively. The CO2 mass balance for all treatments is shown in Figure 1, but not added to the paper.

- I am also concerned that some of the dynamics that we see for NO and N2O gases are solely driven from the closed-nature of the experimental system. For instance, any drop in the amount of NO and N2O in the headspace observed in Figure 2 to 4 and wouldn't really happen in the open system. This type of artificial storage of reactive gases in the headspace interferes with the important storage mechanism of gases within soil aggregates (Rabot et al. 2015; Rabot, Hénault, and Cousin 2014) that significantly affect the total rate of $\mathrm{N} 2 \mathrm{O}$ emission from soil profile (A. Ebrahimi and Or 2018).

Printer-friendly version

Discussion paper 
See response above; it was not the goal of our experiment to disentangle storage of $\mathrm{N}$ gas intermediates in the headspace from $\mathrm{N}$-gas intermediates stored in the pore space of in the pore water. That is the reason why we do not draw any general conclusions from our experiment to open soils under atmospheric conditions. In open systems, it is difficult to quantify how much of the reactive nitrogen has already been converted into N2 or it still present in the soil solution as nitrate or nitrite. Hence, there is a tradeoff between artificiality and measurement uncertainty, which we are currently not able to resolve.

Recommendations: - While my comments may sound rather major, I still believe the study opens up a promising path toward more quantitative understanding of the denitrification process and key players in soil. I think the feature of this study is the quantification of the impact of individual factors (e.g., soil structure, water content, oxygen availability) and offering a well-controlled system with the option of disentangling multiple interacting factors. At this stage, it is fine that the experimental condition does not capture the most common scenarios in natural soil system and it would be insightful if the results would offer generalizable conclusions on the underlying mechanisms. To do this, I recommend that authors put extra work on conceptualizing the role of individual factors on the rates and patterns observed for each of gas fluxes. I think the ideal way would be implementing a mathematical model or at minimum summarizing the results into a conceptual representation of the whole processes.

We have made an attempt now to conceptualize the experimental findings with Fig. 2 and will add this to the Discussion section of the updated manuscript

Interactive comment on Biogeosciences Discuss., https://doi.org/10.5194/bg-2019-2, 2019.

Printer-friendly version

Discussion paper 

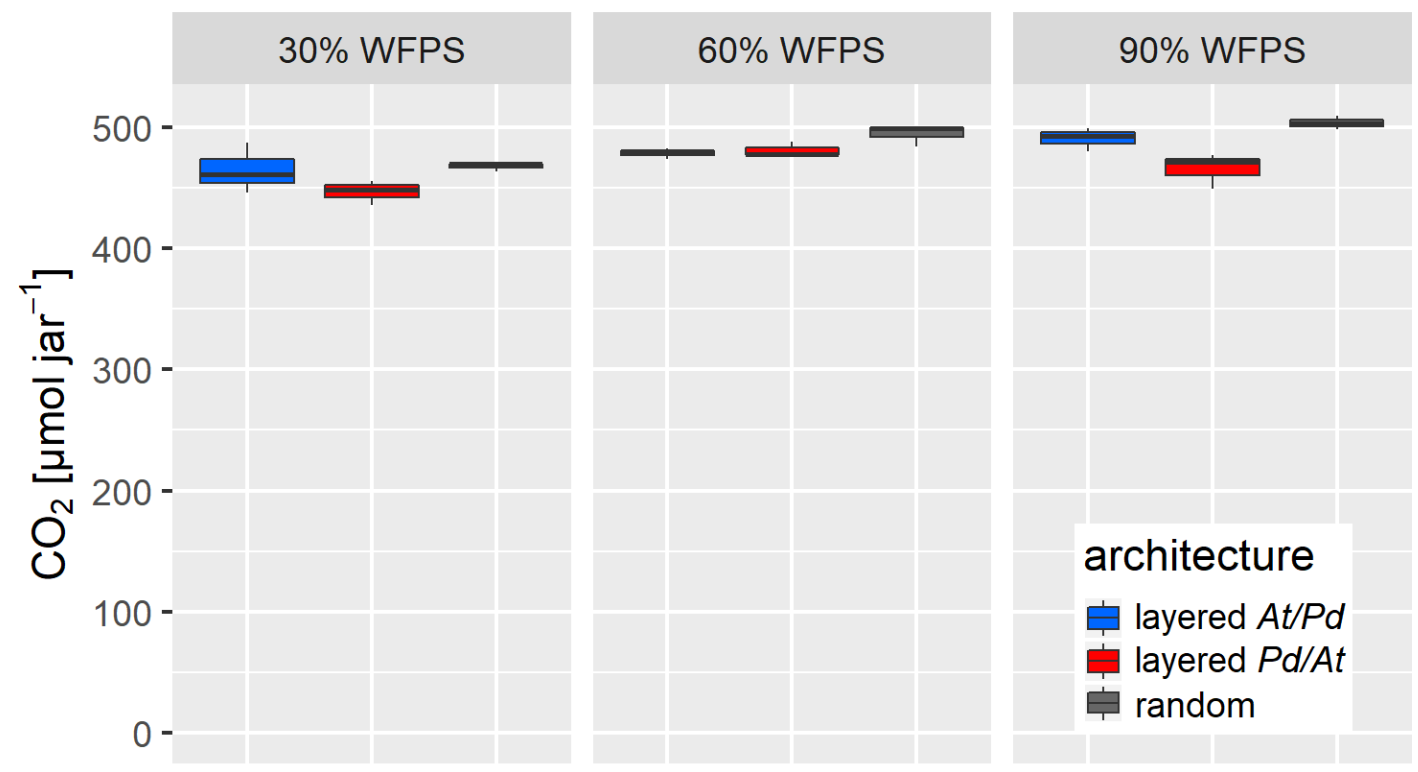

Interactive

comment

Fig. 1. Total $\mathrm{CO} 2$ accumulated in each treatment at the end of incubation $(n=3)$. 
water saturation

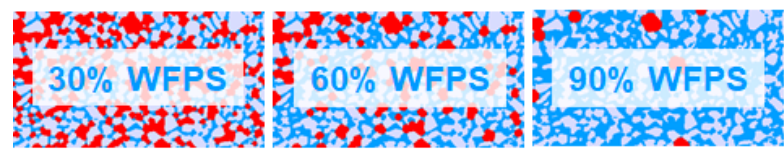

air tortuosity \& distance

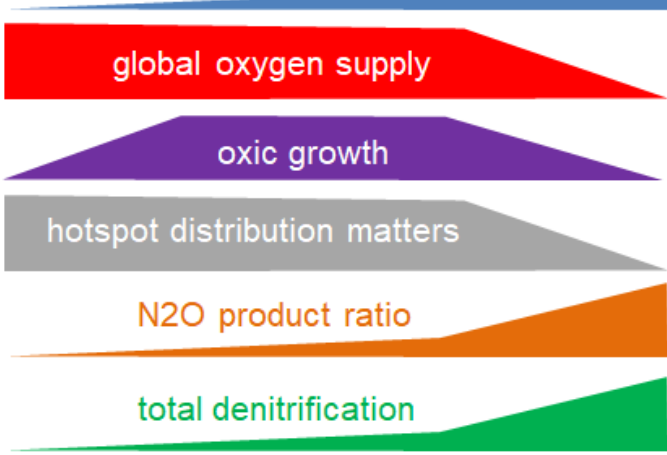

hotspot distribution
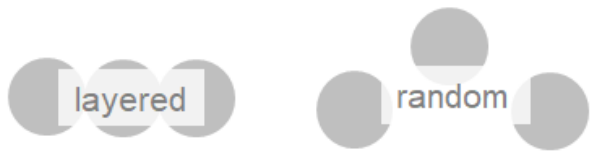

separation distance

local oxygen supply

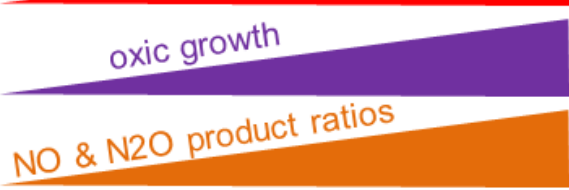

Fig. 2. Conceptual representation of the experimental findings of the incubation setup with a full-factorial design of the factors water saturation and hotspot distribution

Interactive

comment 